\title{
Juntas vecinales y seguridad ciudadana
}

\author{
Mgtr. Claudia Alejandra Cavero Chavez \\ claudia_1312_4@hotmail.com \\ https://orcid.org/0000-0003-4117-1079 \\ Mgtr. Nancy Mariely Ulloa Ale \\ nancy.ulloa@unmsm.edu.pe \\ https://orcid.org/0000-0002-6161-3447
}

Universidad César Vallejo

Lima - Perú

\section{RESUMEN}

El presente artículo científico titulado Juntas Vecinales y seguridad Ciudadana, tiene como objetivo determinar cómo las Juntas Vecinales a través de su formación ayudan a la seguridad ciudadana del distrito, determinar si las acciones son correctas y viables. El presente estudio es de enfoque cuantitativo, teniendo un estudio no experimental, aborda un diseño transversal, busca establecer la observación y mediciones en una muestra, se constituyó una población de 100 miembros de las juntas vecinales del distrito de Paiján, la técnica que se aplicó fue la recolección de datos a través de la encuesta y el instrumento utilizado para esta investigación fue el cuestionario, validado a través de un juicio experto que determinó la fiabilidad mediante la aplicación del Alfa de Cronbach. Se concluyó: La mayoría de los encuestados cree que hay un aumento de la incidencia delictiva también en el distrito de Paiján se han creado juntas vecinales en diferentes sectores, siendo a mediados de 2019 un total de 12 juntas vecinales debidamente conformadas, en relación al año 2020 se crearon un total de 18 juntas vecinales y en 2021 un total de 22 juntas vecinales por otro lado la inseguridad ciudadana aumenta a mayor velocidad. ritmos en relación a los años 2019, 2020 y 2021, teniendo importantes ítems en los que durante esta investigación nos centraremos como las personas requisadas, delito de peligro común y tráfico ilícito de drogas que en base a lo anterior se pueden dar a conocer en la investigación si las juntas vecinales se suman a la tarea de seguridad ciudadana y así disminuye la incidencia penal en la jurisdicción de Paiján.

Palabras clave: gestión; seguridad ciudadana; juntas vecinales; incidencia delictiva. 


\title{
Neighborhood councils and citizen security
}

\begin{abstract}
This research entitled Neighborhood Boards and Citizen Security, determine how neighborhood boards through their formation help citizen security in the district, determine if their actions are correct and viable. The present study is Quantitative approach, a non-experimental study, it also deals with a cross-sectional design, because it seeks to establish the observation and measurements in a sample, about100 members in the neighborhood councils of the district of Paiján was constituted, the technique that applied was the collection of data through the survey and the instrument used for this research was the questionnaire, validated through expert judgment which determined reliability by applying Cronbach's Alpha. It was also concluded: The majority of respondents believe that there is an increase in criminal incidence also in the district of Paiján neighborhood councils have been created in different sectors, being in mid-2019 a total of 12 neighborhood councils duly formed, in relation to the year 2020 a total of 18 neighborhood councils were created and in 2021 a total of 22 neighborhood councils on the other hand the insecurity citizen increases at accelerated paces in relation to the years 2019, 2020 and 2021, having important items which during this investigation we will focus on such as the people requisitioned, crime of common danger and illicit drug trafficking which based on the aforementioned can be made known in the investigation if the neighborhood councils add to the task of citizen security and thus decreases the criminal incidence in the jurisdiction of Paiján.
\end{abstract}

Keywords: management; citizen security; neighborhood boards; crime incidence.

Artículo recibido: 30 noviembre. 2021 Aceptado para publicación: 29 diciembre 2021 Correspondencia: claudia_1312_4@hotmail.com Conflictos de Interés: Ninguna que declarar 


\section{INTRODUCCIÓN}

Las juntas vecinales y su accionar en la seguridad ciudadana asimismo según su accionar y su continuidad se ve reflejado en su viabilidad y continuidad de dichas juntas, actualmente las juntas vecinales sufren de diversas índoles desde la propia participación de los ciudadanos hasta carencia de medios logísticos es por ello que mediante el presente artículo científico se puede dar un aporte a esta labor importante frente a la inseguridad ciudadana la cual arremete contra el país.

La finalidad de las juntas vecinales es apoyar a la gestión de los comisarios y autoridades locales siendo los primeros en enterarse y comunicar cualquier hecho que atente contra la paz y la seguridad de los vecinos y para ello es necesario cumplir con lo establecido según la ley de seguridad ciudadana la cual ampara y protege desde du conformación a las juntas vecinales y mediante resolución de comisaria son conformadas y actúan de acuerdo a sus funciones conforme lo detalla la ley.

Para un buen desempeño y funcionamiento es necesario dotarlos de los implementos necesarios así mismo capacitación continua para poder empoderarlos en base a su función y así puedan desarrollar una labor eficiente la cual sea replica para los demás habitantes de las comunidades en general del Perú, para ello es fundamental que el comisario y el alcalde tengan un contacto directo con los ciudadanos y así ellos vean que sus autoridades son eje fundamental de la paz social que genera ter unidos a los vecinos y hacerlos parte de los diferentes programas preventivos de seguridad ciudadana.

Para el desarrollo de la tesis se formuló como problema general ¿Qué relación existe entre las juntas vecinales y la seguridad ciudadana?, teniendo como objetivo general Determinar la relación de las juntas vecinales y la seguridad ciudadana. Asimismo, se planteó objetivos específicos: Determinar la relación entre el nivel capacitación y la seguridad ciudadana de los ciudadanos del Distrito de Paiján, 2018. Determinar la relación entre el nivel servicio público y la seguridad ciudadana de los ciudadanos del Distrito de Paiján, 2018. Determinar la relación entre el nivel participación y la seguridad ciudadana de los ciudadanos del Distrito de Paiján, 2018.

La justificación de la investigación es argumentar razones que motivan el estudio y tiene propósito definido Julca et al,. (2017), Los resultados obtenidos de la presente investigación servirán como un aporte al conocimiento existente acerca de la formación y el trabajo de las juntas vecinales, identificando las ventajas que las hacen competitivas 
y que inciden en la competitividad operacional de ellas esto quiere decir que servirá como fuente de información para los comisarios a fin de poder implementar un programa productivo del accionar de las juntas vecinales y de esa manera juntos con otros programas puedan desarrollar con éxito su gestión. También se justifica por que incorpora un trabajo de estudio sobre la incidencia delictiva en diferentes jurisdicciones y como las juntas vecinales actúan y cuan preparadas se encuentran lo cual es de carácter objetivo las cuales se denominan como campo de acción asimismo identificando la percepción competitiva de otros comisarios y que tan preparadas están sus juntas vecinales cuantas tienen por zonas y que resultados dan como producción para ello los responsables directos son los alcaldes, comisarios, promotores de la Oficina de Participación Ciudadana (OPC) y capacitadores de la oficina de participación ciudadana.

En la justificación practica Riofrio, L. (2017). en el trabajo de investigación se considera que la presente investigación tiene una justificación práctica, debido a que ayudara a los comisarios quienes tienen a cargo el programa de Juntas Vecinales a tener una mejor perspectiva y ayudara a identificar sus respectivas ventajas competitivas como son el talento humano y el uso de los recursos logísticos a fin de lograr la competitividad durante la gestión del comisario, con la finalidad de obtener productividad durante el trabajo de las juntas, también será una ventana de muestra a fin de diferenciarse de otras comisarias, respecto a la capacitación y accionar esto serán acompañados de los valores identificados como responsabilidad, oportunidad de participación, disciplina y puntualidad. Que servirán como referencia para otros estudios de investigación y para los comisarios en general, así como para los promotores en general para que puedan identificar sus ventajas competitivas y su potencial en recursos humanos.

En la presente investigación se planteó una hipótesis general que: Existe relación significativa entre la junta vecinal y la seguridad ciudadana. Asimismo, se consideró como hipótesis específicas: (i) Existe relación significativa entre la dimensión Capacitación y la seguridad ciudadana. (ii) Existe relación significativa entre la dimensión Servicio Público y la seguridad ciudadana. (iii) Existe relación significativa entre la dimensión Participación y la seguridad ciudadana. 


\section{ESTRATEGIAS METODOLÓGICAS O MATERIALES Y MÉTODOS}

\section{Método}

El presente estudio es no experimental, se trata de un diseño transversal, ya que se busca establecer la observación de variables medidas en una muestra en un único momento del tiempo (Hernández, Fernández, y Baptista; 1991)

\section{Tipo de estudio}

El presente estudio es de enfoque Cuantitativo, como señala García Salinero (2004) son estudios observacionales, en los cuales no se interviene o manipula el factor de estudio, es decir se observa lo que ocurre con el fenómeno en estudio en condiciones naturales, en la realidad. A su vez sabemos que pueden ser clasificados en transversales y longitudinales.

En nuestra investigación será transversal que continuando con García Salinero intentan analizar el fenómeno en un periodo de tiempo corto, un punto en el tiempo, por eso también se les denomina “de corte". Este tipo de estudios pretende identificar y describir fenómenos, mediante la observación y medición de los mismos, intentando dar respuesta a las preguntas básicas (Cleroux \& Jenicek, 1993)

Es aplicada, porque se aplica a una realidad o situación específica, en nuestro caso Juntas vecinales y Seguridad ciudadana en el distrito de Paiján.

\section{Diseño}

Nuestro diseño es descriptivo correlacional transversal; Busca y recoge información relacionada con el objeto de estudio; está constituida por una variable y una población. (Zavaleta,L. 2014)

\section{Esquema:}

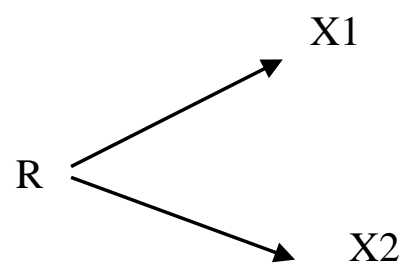

Dónde: $\quad \mathrm{R}=$ Muestra que realizaremos del estudio.

$\mathrm{X}=$ Información relevante o de interés de la muestra.

\section{Población, muestra y muestreo}

\section{Población}

La población estuvo constituida por los habitantes del distrito de Paiján, durante el periodo 2019 - 2021. 


\section{Muestra}

Se realizó un muestreo probabilístico para estimar la proporción, se seleccionó los habitantes con muestreo aleatorio simple, la cual nos dio un tamaño de 120 personas.

\section{Técnicas e instrumentos de recolección de datos}

Técnicas: se empleó como técnica la encuesta; según Naresh K. (2016). Las encuestas son entrevistas con un gran número de personas utilizando un cuestionario prediseñado, dicho cuestionario está diseñado para obtener información específica.

Instrumento: para la investigación se empleó el cuestionario estructurado, la cual consta de 31 preguntas de la Escala Likert.

\section{Validación y confiabilidad del instrumento (s)}

Ambos instrumentos fueron sometidos a pruebas de validez y confiabilidad.

Según Herrera (1998), el instrumento que mide el nivel de la Seguridad ciudadana y juntas vecinales de los pobladores del Distrito de Paiján presenta un Alfa de Cronbach de 0.85, por lo tanto, el instrumento presenta una excelente confiabilidad. Así mismo, el instrumento que mide el nivel de la Seguridad ciudadana y juntas vecinales de los pobladores del Distrito de Paiján presenta un coeficiente de Spearman-Brown de 0.777, por lo tanto, el instrumento es muy válido.

\section{RESULTADOS Y DISCUSIÓN}

\section{Tabla 1}

Distribución entre el nivel de Capacitación y el nivel de Seguridad Ciudadana percibida por los habitantes del distrito de Paiján.

\begin{tabular}{|c|c|c|c|c|c|c|}
\hline & & & Nivel de & guridad & dadana & \\
\hline & & & Bajo & Medio & Alto & Total \\
\hline & Roi & $\mathrm{N}$ & 47 & 4 & 0 & 51 \\
\hline & Вајо & $\%$ & $78,3 \%$ & $22,2 \%$ & $0,0 \%$ & $42,5 \%$ \\
\hline Nivel de & & $\mathrm{N}$ & 13 & 10 & 15 & 38 \\
\hline Capacitación & Medio & $\%$ & $21,7 \%$ & $55,6 \%$ & $35,7 \%$ & $31,7 \%$ \\
\hline & . & $\mathrm{N}$ & 0 & 4 & 27 & 31 \\
\hline & Alto & $\%$ & $0,0 \%$ & $22,2 \%$ & $64,3 \%$ & $25,8 \%$ \\
\hline Totol & & $\mathrm{N}$ & 60 & 18 & 42 & 120 \\
\hline lotal & & $\%$ & $100,0 \%$ & $100,0 \%$ & $100,0 \%$ & $100,0 \%$ \\
\hline
\end{tabular}


Tabla 2. Distribución entre el nivel de Servicio Público y el nivel de Seguridad Ciudadana percibida por los habitantes del distrito de Paiján.

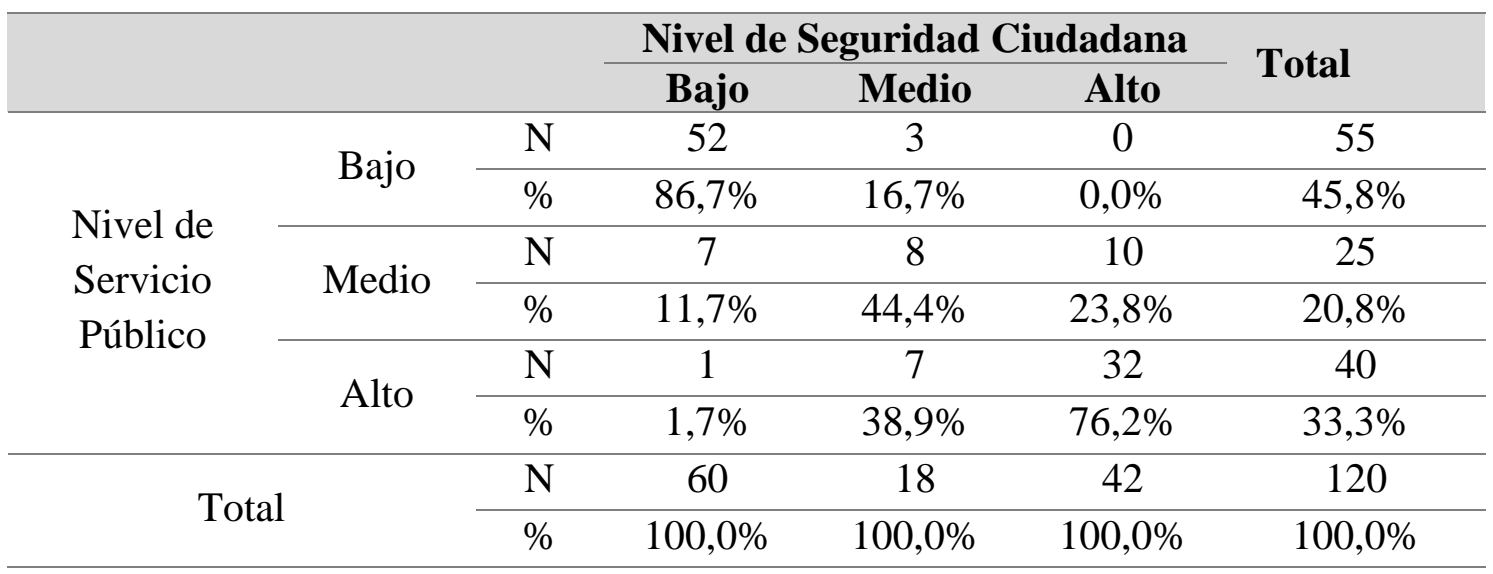

Tabla 3. Distribución entre el nivel de Participación y el nivel de Seguridad Ciudadana percibida por los habitantes del distrito de Paiján.

\begin{tabular}{|c|c|c|c|c|c|c|}
\hline & & & \multicolumn{3}{|c|}{ Nivel de Seguridad Ciudadana } & \multirow{2}{*}{ Total } \\
\hline & & & Bajo & Medio & Alto & \\
\hline \multirow{6}{*}{$\begin{array}{c}\text { Nivel de } \\
\text { Participación }\end{array}$} & \multirow{2}{*}{ Bajo } & $\mathrm{N}$ & 52 & 7 & 2 & 61 \\
\hline & & $\%$ & $86,7 \%$ & $38,9 \%$ & $4,8 \%$ & $50,8 \%$ \\
\hline & \multirow{2}{*}{ Medio } & $\mathrm{N}$ & 3 & 5 & 9 & 17 \\
\hline & & $\%$ & $5,0 \%$ & $27,8 \%$ & $21,4 \%$ & $14,2 \%$ \\
\hline & \multirow{2}{*}{ Alto } & $\mathrm{N}$ & 5 & 6 & 31 & 42 \\
\hline & & $\%$ & $8,3 \%$ & $33,3 \%$ & $73,8 \%$ & $35,0 \%$ \\
\hline \multirow{2}{*}{ Total } & & $\mathrm{N}$ & 60 & 18 & 42 & 120 \\
\hline & & $\%$ & $100,0 \%$ & $100,0 \%$ & $100,0 \%$ & $100,0 \%$ \\
\hline
\end{tabular}

Tabla 4. Distribución entre el nivel de Juntas Vecinales y el nivel de Seguridad Ciudadana percibida por los habitantes del distrito de Paiján.

\begin{tabular}{|c|c|c|c|c|c|c|}
\hline & & & Nivel de & guridad & dadana & T \\
\hline & & & Bajo & Medio & Alto & Total \\
\hline & Roii & $\mathrm{N}$ & 51 & 2 & 0 & 53 \\
\hline & Dajo & $\%$ & $85,0 \%$ & $11,1 \%$ & $0,0 \%$ & $44,2 \%$ \\
\hline Nivel de Juntas & Medis & $\mathrm{N}$ & 7 & 10 & 6 & 23 \\
\hline Vecinales & Miedio & $\%$ & $11,7 \%$ & $55,6 \%$ & $14,3 \%$ & $19,2 \%$ \\
\hline & Alto & $\mathrm{N}$ & 2 & 6 & 36 & 44 \\
\hline & Alto & $\%$ & $3,3 \%$ & $33,3 \%$ & $85,7 \%$ & $36,7 \%$ \\
\hline Total & & $\mathrm{N}$ & 60 & 18 & 42 & 120 \\
\hline 10tal & & $\%$ & $100,0 \%$ & $100,0 \%$ & $100,0 \%$ & $100,0 \%$ \\
\hline
\end{tabular}


Tabla 5: Correlación Rho de Spearman del nivel de Seguridad Ciudadana y nivel de Juntas Vecinales percibido por los habitantes del distrito de Paiján.

\begin{tabular}{|c|c|c|c|c|c|c|c|}
\hline & & & $\begin{array}{l}\text { Nivel de } \\
\text { Seguridad } \\
\text { Ciudadana }\end{array}$ & $\begin{array}{c}\text { Nivel de } \\
\text { Capacitación }\end{array}$ & $\begin{array}{l}\text { Nivel de } \\
\text { Servicio } \\
\text { Público }\end{array}$ & $\begin{array}{c}\text { Nivel de } \\
\text { Participación }\end{array}$ & $\begin{array}{c}\text { Nivel de } \\
\text { Juntas } \\
\text { Vecinales }\end{array}$ \\
\hline \multirow{15}{*}{$\begin{array}{l}\text { Rho de } \\
\text { Spearman }\end{array}$} & \multirow{3}{*}{$\begin{array}{l}\text { Nivel de Seguridad } \\
\text { Ciudadana }\end{array}$} & $\mathrm{R}$ & 1,000 &, $805^{* *}$ &, $848^{* *}$ &, $741^{* *}$ & $859^{* *}$ \\
\hline & & p-valor & . &, 000 &, 000 &, 000 & ,000 \\
\hline & & $\mathrm{N}$ & 120 & 120 & 120 & 120 & 120 \\
\hline & \multirow{3}{*}{ Nivel de Capacitación } & $\mathrm{r}$ &, $805^{* *}$ & 1,000 &, $798^{* *}$ &, $744^{* *}$ &, $837^{* *}$ \\
\hline & & $\mathrm{p}$-valor &, 000 & . &, 000 &, 000 &, 000 \\
\hline & & $\mathrm{N}$ & 120 & 120 & 120 & 120 & 120 \\
\hline & \multirow{3}{*}{ Nivel de Servicio Público } & $\mathrm{r}$ &, $848^{* *}$ &, $798^{* *}$ & 1,000 &, $815^{* *}$ &, $955^{* *}$ \\
\hline & & p-valor &, 000 & ,000 & . &, 000 & ,000 \\
\hline & & $\mathrm{N}$ & 120 & 120 & 120 & 120 & 120 \\
\hline & \multirow{3}{*}{ Nivel de Participación } & $\mathrm{r}$ &, $741^{* *}$ &, $744^{* *}$ &, $815^{* *}$ & 1,000 &, $886^{* *}$ \\
\hline & & $\mathrm{p}$-valor &, 000 &, 000 &, 000 & . &, 000 \\
\hline & & $\mathrm{N}$ & 120 & 120 & 120 & 120 & 120 \\
\hline & \multirow{3}{*}{ Nivel de Juntas Vecinales } & $\mathrm{r}$ &, $859^{* *}$ &, $837^{* *}$ &, $955^{* *}$ &, $886^{* *}$ & 1,000 \\
\hline & & $\mathrm{p}$-valor &, 000 &, 000 &, 000 &, 000 &, 000 \\
\hline & & $\mathrm{N}$ & 120 & 120 & 120 & 120 & 120 \\
\hline
\end{tabular}

**. La correlación es significativa en el nivel 0,01 (bilateral).

En la Tabla 1 podemos observar que de los habitantes del distrito de Paiján que percibió el nivel de Seguridad Ciudadana bajo, el 78.3\% posee un nivel de Capacitación Bajo y el 21.7\% un nivel medio; de los que percibieron nivel de Seguridad Ciudadana Medio, un $22.2 \%$ presentó un nivel bajo, así mismo como nivel alto y $55.6 \%$ nivel medio; finalmente los que percibieron nivel alto de seguridad ciudadana, $35.7 \%$ tuvo un nivel medio en capacitación y $64.3 \%$ nivel alto, dichos datos tienen una relación estrecha con el trabajo de Weiffen (2017), donde se tiene una conclusión la cual guarda relación estrecha relación entre la variable seguridad ciudadana y participación de las juntas vecinales teniendo una correlación de variables de 0,857 y altamente significativa con un valor $=0,000<0,01$. Del mismo modo Mirano (2019), manifiesta que la seguridad ciudadana implica una programación de diversas actividades las cuales se ejecuten y se comprometan a apoyar en la prevención del delito, teniendo un apoyo del comisario del sector y con capacitación continua, otro punto importante es la unión de las variables las cuales guardan relación, siendo una conclusión general prevenir los problemas de seguridad ciudadana. 
Mientras tanto, Rodríguez (2014), Nos menciona que los problemas de inseguridad ciudadana no solo radican en la falta de efectivos policiales en las calles y las deficientes autoridades en el Ministerio Publico, si no que parte de un aporte es una comunidad organizada la cual cumple un papel principal primario de la misma manera que lo cumplen los gobiernos locales y departamentales. Según la teoría de Dávila (2010), Los problemas de inseguridad ciudadana han incrementado debido a una mala utilización de recursos por parte de las autoridades locales y es por ello que se ve reflejado durante las diferentes gestiones y ello recae en la minúscula participación de los ciudadanos en diversas actividades ello se refleja en el interés en su comunidad y esas normas de conductas se hacen extensivas tomándolo como un estilo convivencia social normal, para combatir lo antes expuesto por el autor aún no hay una unión de esfuerzos para buscar una solución positiva para los ciudadanos.

La Tabla 2 podemos observar que de los habitantes del distrito de Paiján percibieron un nivel de Seguridad Ciudadana bajo, el 86.7\% manifestó un nivel de Servicio Público Bajo, de un nivel medio el $11.7 \%$ y solo el $1.7 \%$ un nivel alto; de los que percibieron nivel de Seguridad Ciudadana Medio, un $16.7 \%$ presentó un nivel bajo, así mismo como nivel medio el $44.4 \%$ y $38.9 \%$ nivel alto; finalmente los que percibieron nivel alto de seguridad ciudadana, en servicio público bajo representa el $45.8 \%$; $20.8 \%$ obtuvo un nivel medio y $33.3 \%$ nivel alto en servicio público, también el punto de vista de Trujillo (2021), Que las juntas vecinales deben ser fortalecidas y capacitadas. Al mismo tiempo Riofrio (2017), En su investigación demostró arduamente que las juntas vecinales son un gran apoyo a la ciudadanía y que ellas contribuyen un estado de paz encargándose de la tranquilidad de los habitantes y así contribuyen a una seguridad sostenible la cual debe de perdurar y así ser replica para los futuros habitantes y ello se logra con capacitación y esfuerzo constante de las autoridades.

La Tabla 3 podemos observar que de los habitantes del distrito de Paiján percibieron un nivel de Seguridad Ciudadana bajo, el $86.7 \%$ manifestó un nivel de Participación Bajo, de un nivel medio del $5 \%$ y un $8.3 \%$ un nivel alto; de los que percibieron nivel de Seguridad Ciudadana Medio, un 38.9\% presentó un nivel bajo, así mismo como nivel medio el $27.8 \%$ y $33.3 \%$ nivel alto de Participación; finalmente los que percibieron nivel alto de Seguridad Ciudadana, en participación bajo representa el 50.8\%; 14.2\% obtuvo un nivel medio y $35 \%$ nivel alto de participación. Este resultado tiene como soporte lo 
manifestado por Tapia (2020), Donde manifiesta que para disminuir la inseguridad ciudadana los funcionarios deben enfocarse en tener una buena estructura organizacional entre las entidades policiales y municipios y ello se complementara con la proactividad de los ciudadanos mediante programas preventivos como son las juntas vecinales, estos programas ayudaran a cooperar con la seguridad pero a la vez el autor nos plasma que es importante la capacitación continua comparándolo con los colaboradores de una organización donde si no hay capacitación continua no hay mejoras en sus procesos a corto o largo plazo.

Mientras tanto Grozzo (2019), El autor manifiesta que la PNP y autoridades de la localidad son responsables del porque no se practica las juntas vecinales con los ciudadanos calificándolo como falta de compromiso para con su localidad, para ello se empodera a los ciudadanos para que puedan agruparse y tener voz para poder unificar esfuerzos y puedan unirse al desarrollo con el objetivo de generar confianza e identidad en los demás habitantes ante los problemas de inseguridad.

Como expresa Mattos (2015), Nos refiere que toda organización debe de tener subniveles los cuales se deben encargar de fomentar programas que unan a los habitantes y deleguen responsabilidades agrupando actividades los cuales se plasmen en los objetivos trazados. En la Tabla 4 podemos observar que de los habitantes del distrito de Paiján que percibió el nivel de Seguridad Ciudadana bajo, el 85\% manifestó un nivel de Juntas Vecinales Bajo, nivel medio un $11.7 \%$ y solo el $3.3 \%$ un nivel alto; de los que percibieron nivel de Seguridad Ciudadana Medio, un $11.1 \%$ presentó un nivel bajo, así mismo en nivel medio un 55.6\% y $33.3 \%$ nivel alto de juntas vecinales; finalmente los que percibieron nivel alto de seguridad ciudadana, $14.3 \%$ tuvo un nivel medio en juntas vecinales y $85.7 \%$ nivel alto. Según Diez (2017), La seguridad se basa en una cultura de paz donde los vecinos puedan vivir en armonía siendo un bien público que así lo demanda la sociedad y cada ciudadano tiene un rol específico para una contribución a la misma siendo de esa manera un ente replicador de buenas costumbres practicadas mediante las medidas preventivas como son las juntas vecinales siendo una conducta positiva que combina paz y armonía entre los habitantes para ello es necesario tener políticas públicas de seguridad ciudadana eficientes y trabajadas al detalle para que sea viable. Sánchez (2019), Demuestra que los funcionarios públicos no están capacitados por los gobiernos regionales y locales siendo 
administradores de crisis regionales y locales en vez de regular y capacitar a su personal para que puedan trabajar con los habitantes y capacitarlos para que sean lideres.

La tabla 5 nos muestra la matriz de correlaciones bivariadas, en ella se muestra el coeficiente de correlación Rho de Spearman y su significancia estadística después de haber sido sometida a la prueba de hipótesis respectiva; la primera columna es la que nos proporciona los resultados necesarios para poder responder a los objetivos planteados y contrastar las hipótesis, en ella se manifiesta la correlación de pares de variables, la variable Seguridad Ciudadana con la variable Juntas vecinales, así como las dimensiones de esta última. En todos los pares de variables se encontró relación altamente significativa (p-valor < 0.01), es decir que existe relación entre Seguridad Ciudadana y Juntas Vecinales, como también con las dimensiones Capacitación, Servicio Público y Participación; la relación entre Seguridad ciudadana y Juntas vecinales es directa junto con sus dimensiones, hubo una relación fuerte con Juntas vecinales (0.859) y sus dimensiones: Capacitación (0.805), Servicio Público (0.848) y Participación (0.741). Mattos (2015), En su investigación concluye que las políticas públicas tienen por objeto reducir los altos índices de inseguridad ciudadana en los lugares más alejados del país y para ello es fundamental empoderar a los habitantes de las comunidades par que ellos ante una ausencia de sus autoridades ellos pueden tener un control permanente sobre diferentes acciones que se puedan tomar para el bien de la comunidad mitigando y previniendo la inseguridad ciudadana.

\section{CONCLUSIÓN O CONSIDERACIONES FINALES}

Primera: La relación entre el nivel de capacitación y seguridad ciudadana de los ciudadanos del distrito de Paiján fue significativa ( $\mathrm{p}$ valor $<0.05$ ), con una correlación de 0.805 , existiendo relación positiva fuerte.

Segunda: La relación entre el nivel de servicio público y seguridad ciudadana de los ciudadanos del distrito de Paiján fue significativa ( $\mathrm{p}$ valor $<0.05$ ), con una correlación de 0.848 , existiendo relación positiva fuerte.

Tercera: La relación entre el nivel de participación y seguridad ciudadana de los ciudadanos del distrito de Paiján fue significativa ( $\mathrm{p}$ valor $<0.05$ ), con una correlación de 0.741, existiendo relación positiva fuerte. 
Cuarta: Se llegó a la conclusión general que la relación entre seguridad ciudadana y juntas vecinales fue significativa ( $\mathrm{p}$ valor $<0.05$ ), con una correlación de 0.859 , existiendo relación positiva fuerte.

\section{LISTA DE REFERENCIAS}

Acosta Vásquez, R. J. (2019). Participación de las juntas vecinales en el desarrollo local del distrito de Guadalupe durante el período 2017-2018. https://dspace.unitru.edu.pe/handle/UNITRU/14700

Aguirre, H. M. (2019). Juntas Vecinales y la toma de decisiones municipales eneldistritodeTumbes-2016.

http://repositorio.untumbes.edu.pe/handle/20.500.12874/273

Ayala, M., \& Quisbert, A. (2019). Rol de las juntas vecinales en el fortalecimiento de la seguridad ciudadana del distrito de Aucallama-2018. http://200.48.129.167/handle/UNJFSC/2595

Bailes et al,. (2006), Regional Security cooperation in the early 21st century in Stockholm International Peace Research Institute. SIPIRI Yearbook 2006: Armaments Disarmament and International Security. Oxford University Press:Oxford,pp.195223. https://www.sipri.org/sites/default/files/YB06\%20195\%2004.pdf .

Barrio et al,. (2016), "improving Comprehension of Numbers in the News," paper presented at the Association for Computing Machinery Conference on Human Factors in Computing Systems, San Jose, CA (May 7-12). https://dl.acm.org/doi/abs/10.1145/2858036.2858510

Calderón, L., \& Ramírez, R. (2018). Seguridad ciudadana y juntas vecinales enSanBartolome,distritodeSantaMaría,2016. http://200.48.129.167/handle/UNJFSC/3556

Chavez Vasquez, K. D. (2017). Regulación de un mecanismo de control en las juntas vecinales. http://repositorio.upao.edu.pe/handle/20.500.12759/3089

Dávila, D. (2010). Las Juntas vecinales en el fortalecimiento de la seguridad ciudadana del distrito de San Juan de Lurigancho, del 2006 hasta el 2009. http://cybertesis.unmsm.edu.pe/handle/20.500.12672/3309

Díaz Carranza, Á. S. (2018). Seguridad ciudadana, y la acción de las juntas vecinales distrito de Carabayllo, periodo 2016 al 2017. https://core.ac.uk/download/pdf/225606079.pdf 
Díaz Sánchez, J. F. (2019). Gestión de seguridad ciudadana y la participación de las juntas $\begin{array}{lllll}\text { vecinales en distrito el de } & \text { Bellavista, }\end{array}$ https://core.ac.uk/download/pdf/232928685.pdf

Elcorrobarrutia, R. (2020). La participación comunitaria en relación con el derecho a la seguridad ciudadana en el distrito de Chancay, provincia de Huaral,regiónLima,2018. https://repositorio.upn.edu.pe/handle/11537/24393

Emerson, R. G. (2020). Who Is the Citizen in Citizen Security? Latin American Research Review, 55(3), 529-543. DOI: http://doi.org/10.25222/larr.454

Gasper, D. and Gómez, O. (2015). Human security thinking in practice: 'personal security', 'citizen security' and comprehensive mappings, Contemporary Politics, 21:1, 100-116, DOI: 10.1080/13569775.2014.993906

Goldstein, D. (2015), Citizen Security and human security in Latin America In D R Mares of Latin American Security. Research Review 11 1st Edition. London: Routledge. https://doi.org/10.4324/9781315867908

Grozzo, C. (2019). Participación activa de la ciudadanía en la labor policial y fiscal, para hacer frente a la inseguridad ciudadana respecto a los delitos contra el patrimonio, robo y hurto: año 2016 en la ciudad de Chiclayo. http://tesis.pucp.edu.pe/repositorio/handle/20.500.12404/15450

Heine, J. y Weiffen, B. (2015), "The challenges of regional democracy promotion. Century Democracy Promotion in the Americas: Standing Up for the new Polity. New York. Pp. 10-29”. https://www.routledge.com/21st-Century-DemocracyPromotion-in-the-Americas-Standing-up-for-the-Polity/HeineWeiffen/p/book/9780415626378

Julca et al,. (2017), Rondas campesinas y nativas en el ejercicio de la seguridad ciudadana de la región de San Martin. Revista Ciencia y Tecnología,13(2),79-89. https://revistas.unitru.edu.pe/index.php/PGM/article/view/1862

Marcella, G. (2013). The Transformation of Security in Latin America: Cause for Common Action. Journal of International Affairs 67-82. https://www.jstor.org/stable/24388286 
Martínez, M. (2019). Logros y desafíos de la integración centroamericana: aportes de la $\begin{array}{lllllll}\text { CEPAL, Libros de la CEPAL, } & \mathrm{N}^{\circ} 156 \quad \text { (LC/PUB.2019/7- }\end{array}$ P).https://www.sica.int/download/?119076

Marulanda, C., López, M., \& López, F. (2016). La cultura organizacional y las competencias para la gestión del conocimiento en las pequeñas y medianas empresas (pymes) de Colombia. Información tecnológica, 27(6), 03-10.” https://scholar.google.com.pe/scholar?q=L\%C3\%B3pez,+M.+(2016)\&hl=es\&as_sd $\mathrm{t}=0$ \&as_vis $=1 \&$ oi $=$ scholart

Mattos, A. (2015). La seguridad ciudadana bajo el enfoque de la seguridad humana. https://repositorioacademico.usmp.edu.pe/handle/20.500.12727/2646

Medina, J, Becerra, S., \& Castaño, P. (2014). Prospectiva y política pública para el cambio estructural en América Latina y el Caribe. CEPAL. Prospectiva y política pública para el cambio estructural en América Latina y el Caribe, Libros de la CEPAL, N 129 (LC/G.2622-P), Santiago de Chile, Comisión Económica para $\begin{array}{llll}\text { América Latina } & y & \text { Caribe (CEPAL). }\end{array}$ https://repositorio.cepal.org/handle/11362/37057

Morlino, L. (2015). Qualidades da democracia: como analisá-las Sociedade e Cultura, vol. 18, núm. 2, julio-diciembre, 2015, pp. 177-194. Universidade FederaldeGoiásGoiania,Brasil.

https://www.scielo.br/j/ccrh/a/qJc4qDsjqyYNTyPtGHm9Shm/abstract/?lang=pt

Nery, T, (2016). UNASUR: A dimensão política do novo regionalismo sudamericano.Caderno,CRH29(3):59-75.

https://www.scielo.br/pdf/ccrh/v29nspe3/0103-4979-ccrh-29-spe3-0059.pdf

Oviedo Maravi, L. (2010). Plataforma de Monitoreo Vecinal para enfrentar la deficiente gestión de la información sobre las Juntas Vecinales de Seguridad Ciudadana en el distrito de Cercado de Lima. http://tesis.pucp.edu.pe/repositorio/handle/20.500.12404/19458

Pries, Kari. (2017). Contested concepts and practices in security governance: evolving security approaches in El Salvador. $\mathrm{PhD}$ thesis, University of Glasgow. https://theses.gla.ac.uk/8070/

Riofrio, L. (2017). La incorrecta aplicación de la institución del arresto ciudadano por parte de las juntas vecinales encargadas de la seguridad ciudadana en el distrito 
$\begin{array}{lllll}\text { judicial de } & \text { la } & \text { Banda }\end{array}$ https://repositorio.uss.edu.pe/handle/20.500.12802/4689

Ríos, C., \& Mussoline, A. (2019). Participación Ciudadana de las Juntas Vecinales en las Políticas Públicas Preventivas de Seguridad Ciudadana del DistritodeMiraflores, Arequipa2018. http://tesis.ucsm.edu.pe/repositorio/handle/UCSM/8818

Rodríguez, F. (2014). La co-enseñanza, una estrategia para el mejoramiento educativo y la inclusión. Revista latinoamericana de educación inclusiva, 8(2), 219-233. https://dialnet.unirioja.es/descarga/articulo/4994333.pdf

Romero, J. (2010). Evolución institucional de la seguridad en el marco del sistema de la integración centroamericana. Buenos Aires. 1a. Ed. - Córdoba, España. : Fundación ETEA para el Desarrollo y la Cooperación, 2010. https://www.sica.int/download/?44877

Sánchez, C. (2019). Participación Ciudadana en el Presupuesto Participativo y la Democracia en las Juntas Vecinales, Región de Puno-2017. https://alicia.concytec.gob.pe/vufind/Record/2521-

\section{7_0681da5e39f9838fc91687b0aa140259}

Santa Cruz, D. (2010). Las juntas vecinales como mecanismo de seguridad ciudadana con éxito en los pobladores de los barrios de la ciudad de Puno-2008. http://tesis.unap.edu.pe/handle/UNAP/759

Tickner, A.B and Herz, M. (2012) No place for theory: Security studies in Latin America. In AB. New York: Routledge. Pp. 92-114. https://www.academia.edu/27928866/No Place for Theory Security Studies in Latin America

Torres, B., \& Cuchuirumi, D. (2018). Funcionamiento del sistema de seguridad ciudadana desde el gobierno local y las juntas vecinales en la ciudad de Puno, 2017. http://repositorio.unap.edu.pe/handle/UNAP/11352

Trujillo, J. (2021). Las juntas vecinales y seguridad ciudadana en el AA. HH. AtalayadeldistritodeHuacho2019.

http://repositorio.unjfsc.edu.pe/handle/UNJFSC/4580

Weiffen, R. (2017). Re -thinking Latin American Regional Security: The impact of power and politics. Research Review. https://www.academia.edu/36723589/Regionalism with Adjectives in Latin America book review 